\title{
Thyroid disorders in alemtuzumab-treated multiple sclerosis patients: a Belgian consensus on diagnosis and management
}

\author{
Brigitte Decallonne ${ }^{1}$ (D) Emmanuel Bartholomé ${ }^{2} \cdot$ Valérie Delvaux $^{3} \cdot$ Miguel D'haeseleer $^{4,5} \cdot$ Souraya El Sankari ${ }^{6}$. \\ Pierrette Seeldrayers ${ }^{7} \cdot$ Bart Van Wijmeersch $^{8} \cdot$ Chantal Daumerie $^{9}$
}

Received: 9 October 2017 / Accepted: 15 January 2018 / Published online: 25 January 2018

(c) The Author(s) 2018. This article is an open access publication

\begin{abstract}
This paper deals with thyroid disease that can occur after treatment with alemtuzumab (humanized monoclonal anti-CD52) for relapsing-remitting multiple sclerosis (MS). The 5-year incidence of thyroid adverse events in phase 3 clinical trials is up to $40.7 \%$. In most cases, the thyroid dysfunction is mild and easily manageable and only few serious thyroid adverse events have been reported. The need for patient education on the risk of thyroid dysfunction, as well as regular clinical and biochemical thyroid function screening is well described. However, practical clinical guidance in case of abnormal thyroidrelated findings prior to or after alemtuzumab treatment is currently lacking. Therefore, a Belgian taskforce consisting of MS and thyroid experts was created in 2016, with the objective of issuing a clinical thyroid management algorithm based on available scientific evidence and personal experience with regard to alemtuzumab treatment-related thyroid adverse events.
\end{abstract}

Keywords Multiple sclerosis · Alemtuzumab $\cdot$ Autoimmune thyroid disease $\cdot$ Immune reconstitution $\cdot$ Algorithm . Management

Brigitte Decallonne

brigitte.decallonne@uzleuven.be

1 Department of Endocrinology, Universitair Ziekenhuis Gasthuisberg, Herestraat 49, 3000 Leuven, Belgium

2 Department of Neurology, CHU Tivoli, La Louvière, Belgium

3 Department of Neurology, CHU de Liège, site CHR, Liège, Belgium

4 Department of Neurology, Universitair Ziekenhuis Brussel, Brussels, Belgium

5 Nationaal MS Centrum, Melsbroek, Belgium

6 Department of Neurology, Cliniques Universitaires St Luc, Brussels, Belgium

7 Department of Neurology, CHU de Charleroi, Charleroi, Belgium

8 Department of Neurology, Rehabilitation and MS Centre, Overpelt, Belgium

9 Department of Endocrinology, Cliniques Universitaires St Luc, Brussels, Belgium

\section{Introduction}

Alemtuzumab is a humanized monoclonal antibody approved for the treatment of adult patients with active multiple sclerosis (MS) in more than 65 countries [1, 2]. In clinical trials, alemtuzumab demonstrated superior efficacy compared to an active comparator (subcutaneous interferon beta-1a) in both treatment-naive patients and in those with inadequate response to prior therapy [3-5]. The mechanism by which alemtuzumab exerts its therapeutic effects in MS is not fully elucidated. However, research suggests immunomodulatory effects through the depletion of mature and circulating B- and $\mathrm{T}$ lymphocytes, followed by a repopulation and long-lasting shift in immunological balance, including alterations in the number, proportions, and properties of some lymphocyte subsets, increased representation of regulatory T-lymphocyte subsets, and increased representation of memory T- and B lymphocytes. This mechanism may be relevant to the durable efficacy of this drug [6-8]. Treatment may result in the formation of autoantibodies and increase the risk of autoimmune mediated adverse events. Autoimmune thyroid disease (AITD) has been the most common autoimmune disorder, followed by immune thrombocytopenia (ITP), and nephropathies. According to a hypothesis, differential kinetics of lymphocyte subset 
reconstitution causing early hyperrepopulation of immature B lymphocytes may explain the occurrence of autoimmune events [9]. A risk management plan (RMP) has been implemented to mitigate the risk of autoimmune conditions in MS patients treated with alemtuzumab. Thyroid function tests, such as serum thyroid stimulating hormone (TSH) levels, should be obtained prior to initiation of treatment and every 3 months thereafter until 48 months following the last infusion. After this period, testing should be performed based on the clinical findings suggestive of thyroid dysfunction. Systematic thyroid function monitoring was integrated as of the phase 2 clinical trial [3]. In the phase 3 clinical trials and the extension study, 40.7\% (CARE-MS I) and 37.7\% (CARE-MS II) of alemtuzumab-treated subjects developed thyroid adverse events (TAEs) within 5 years, more precisely thyroid dysfunction, with a peak incidence in the third year following the first administration [10-13]. In the event of abnormal findings, the treating physician will have to decide how to manage the sequence of additional tests, possible treatments, and the need to refer the patient to an endocrinologist for further treatment and follow-up. Post-marketing surveillance figures have not been published so far. Due to the current lack of practical clinical guidance with regard to managing thyroid-related autoimmunity after alemtuzumab treatment, a Belgian taskforce was created in 2016 consisting of experts in MS and in thyroid disease, with the objective of issuing practical recommendations to guide the treating physician.

\section{Methodology}

A Belgian taskforce, consisting of 6 experts in MS (E.B., V.D., M.D., S.E., P.S., B.V.) and 2 experts in thyroid disease (B.D., C.D.) first met on May 23, 2016, with the objective of discussing thyroid autoimmunity related to treatment with alemtuzumab based on scientific evidence and personal experience, and designing a clinical management algorithm. During this meeting, the results from the phase 3 CARE-MS I and II and the extension study were discussed along with the major practice points. Next, a draft clinical algorithm was created, which was further developed and discussed through electronic correspondence among the experts, eventually resulting in a consensus clinical algorithm falling into 2 parts: thyroid-related management prior to and post administration of alemtuzumab.

\section{Autoimmune thyroid disease in the general population}

AITD represents the most common autoimmune disease with an estimated prevalence of $5 \%$, and is more frequent in females [14]. The risk of AITD is further increased in the presence of other autoimmune disease(s), such as MS
[15]. Usually, AITD presents as 1 of 3 classical phenotypes: autoimmune hypothyroidism or Hashimoto's disease (HD), autoimmune hyperthyroidism or Graves' disease (GD), or silent thyroiditis (ST). AITD, however, has to be considered as a dynamic spectrum of diseases, including non-classical phenotypes.

HD is the most common phenotype of AITD. It is a form of painless chronic lymphocytic thyroiditis, and usually slowly develops over years to decades, eventually and most frequently irreversibly leading to hypothyroidism due to thyroid tissue destruction. In most cases, serum thyroperoxidase (TPO) antibodies (Abs) are increased. The treatment consists of lifelong synthetic thyroxine (levothyroxine; LT4) supplementation [16, 17].

GD represents the second most common AITD phenotype, usually developing over weeks to months. GD results in hyperthyroidism, caused by uncontrolled stimulation of the TSH or thyrotropin receptor (TR) by thyrotropin receptor antibodies (TRAbs). TRAbs are thus pathogenic by causing thyroid gland stimulation and thyroid hormone overproduction. Increased TRAb titers represent the hallmark of GD. Rarely, neutral or even blocking TRAbs develop instead of stimulating TRAbs [18]. TPOAbs can also be increased and define the signature of AITD. GD treatment is more complex and consists of reversible medical treatment for 12-18 months with thionamides, anti-thyroid drugs (ATDs) interfering with the TPO enzyme and thus thyroid hormone synthesis, or irreversible non-surgical (radioactive iodine) or surgical (thyroidectomy) treatment $[19,20]$. The treatment choice is the result of shared clinical decision between the endocrinologist and the patient. Usually, ATD treatment is well tolerated. However, there are rare cases of severe side-effects, such as agranulocytosis, vasculitis, and liver dysfunction [21]. GD can also target the retro-orbital fat and muscle tissue, resulting in Graves' orbitopathy (GO), characterized by unilateral or bilateral asymmetrical redness, swelling, diplopia, and very rarely dysthyroid optical neuropathy. The risk of GO is increased with severe hyperthyroidism, high TRAbs, smoking, and in males [22].

ST, the third classical AITD phenotype, is characterized by a self-limiting painless subacute lymphocytic thyroiditis, lasting for weeks to months. TPOAbs are often increased. In the first phase, increased thyroid hormone levels can be observed due to leakage caused by thyroid inflammation, whereas transient hypothyroidism can develop in the second phase. Eventually thyroid function is restored [23].

Thyroid dysfunction and AITD are associated with increased risk for infertility, obstetrical complications, and neuropsychological problems in the offspring [24]. Women with known AITD who are attempting to become pregnant need to be informed of the risks by the endocrinologist and gynecologist. Euthyroidism is mandatory pre-conception. In pregnant women treated with ATDs and/or having persistently 
high TRAbs, fetal or neonatal hypo- and hyperthyroidism may develop [25].

The diagnosis of AITD is made by measurement of serum thyrotropin or TSH (primary screening parameter for any thyroid dysfunction), thyroid hormones, and antibodies (TPOAbs in case of hypothyroidism, TRAbs in case of hyperthyroidism). If a hyperthyroid patient is symptomatic, nonspecific beta-blockade (propranolol) is indicated independent of the underlying cause. In case of GD and ST, and in case of clinical thyroid gland abnormalities (e.g., goiter), anatomical and functional imaging is indicated by means of ultrasonography and scintigraphy (with radioactive technetium or iodine as tracer), respectively. In a hyperthyroid patient, decreased tracer uptake is suggestive of ST, whereas normal or increased uptake is suggestive of GD [16, 17, 19, 20].

\section{Autoimmune thyroid disease post-alemtuzumab}

AITD is the most frequently observed autoimmune adverse event in MS patients treated with alemtuzumab [3-5, 26]. TAEs can develop from 6 months post-alemtuzumab onward and have a peak incidence in year 3 after the first course and a gradual decline afterwards $[10,11]$. In clinical trials, half the patients who tested positive for TPO Ab at baseline and a quarter of patients who tested negative developed a thyroid event. The vast majority (approximately $80 \%$ ) of patients who presented with a thyroid event after treatment were TPO $\mathrm{Ab}$ negative at baseline [27]. In contrast to the general population, AITD after alemtuzumab presents 4 times more often as hyperthyroidism than as hypothyroidism. The thyroid dysfunction is usually mild to moderate, and easily controlled with conventional therapy. Mild cases can resolve spontaneously. Serious TAEs such as GO, neonatal GD, and psychiatric events were rare (1-2\%) [28-31]. Furthermore, GD tends to spontaneously resolve in around 1 of 3 cases, which could be due to a higher occurrence of neutral or blocking instead of the classical stimulating TRAbs. Therefore, AITD post-alemtuzumab seems to clinically present as rather 'nonclassical' phenotypes, also reported in other immune reconstitution circumstances such as HIV treatment, and might, therefore, require a different treatment approach compared to AITD in the general population [32, 33]. Furthermore, the occurrence of thyroid dysfunction did not affect quality of life of alemtuzumab-treated MS patients [34-36].

\section{Proposal for thyroid-related management prior to the initiation of alemtuzumab}

Before deciding to treat an eligible patient with alemtuzumab, 2 practical steps should be undertaken with regard to risk assessment for thyroid dysfunction. First, attention should be paid to past or current risk factors known to interfere with thyroid function, including medication and smoking behavior [37]. Second, careful interpretation of the mandatory baseline thyroid tests is needed along with adequate action in case of abnormal findings. The schematical overview of the proposed pre-alemtuzumab clinical management algorithm is shown in Fig. 1.

\section{Baseline screening}

It is important to ask about previous or current problems with thyroid function. In case of current treatment with LT4 for hypothyroidism, measurement of TSH and TPOAb status is indicated to check for the correct supplementation dose and for the nature of the hypothyroidism. In case of current or recent treatment for hyperthyroidism (any cause), current or past GD, known intolerance to ATDs, or the presence of a clinical thyroid abnormality (e.g., a thyroid nodule), the patient should first be referred to an endocrinologist for additional thyroid management, and a shared clinical decision needs to be made with regard to delay of or contraindication for the initiation of alemtuzumab. Finally, ask about intentions with regard to pregnancy desire when dealing with female patients of childbearing age.

\section{Management in case of abnormal findings}

In case of a negative thyroid history and a normal TSH, treatment with alemtuzumab can be started. When a patient is currently treated with LT4 and TSH is abnormal, proper adaptation of the LT4 dose is needed along with a re-evaluation of serum TSH after (minimally) 6 weeks. In the meantime, alemtuzumab can be started without delay.

In case of increased serum TSH at baseline screening, additional free T4 (fT4) testing is indicated. When free T4 levels are decreased, indicative of hypothyroidism, LT4 supplements should be started. When free T4 levels are normal, indicative of 'subclinical' hypothyroidism, LT4 treatment can be considered and alemtuzumab can be started.

In case of positive TPOAbs in the patient with or without LT4 supplementation, there is an increased risk for the development of TAEs but alemtuzumab can be started.

When decreased TSH is observed, additional testing of fT4 and TRAbs is indicated, and treatment with propranolol should be started in case of symptoms or signs of hyperthyroidism or a fT4 1.5-fold above the upper limit of normal (ULN). Next, the patient needs to be referred to an endocrinologist for additional thyroid management along with shared clinical decision making on the initiation of alemtuzumab. 


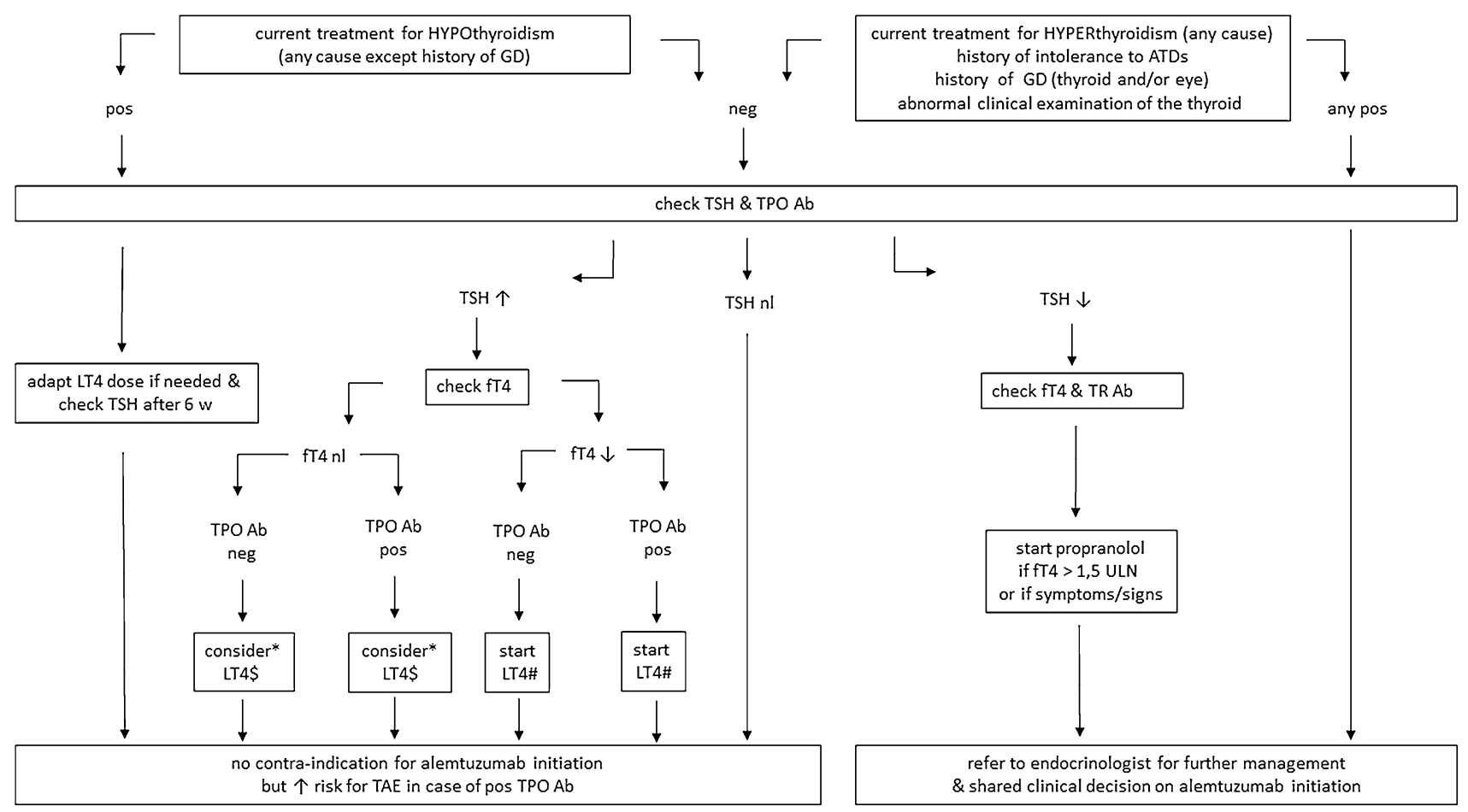

Fig. 1 Thyroid management algorithm prior to alemtuzumab. $A b$ antibody, ATD anti-thyroid drug, fT4 free T4, LT4 levothyroxine, neg negative, $n l$ normal, pos positive, TAE thyroid adverse event, TPO thyroperoxidase, $T R$ thyrotropin receptor, $T S H$ thyroid stimulating hormone, $U L N$ upper limit of normal. *Symptoms/signs of hypothyroidism or TSH $>10 \mathrm{mU} / \mathrm{L}$ favor initiation of LT4; ${ }^{\$} \mathrm{LT} 4 \pm 0.5 \mu \mathrm{g} /$ $\mathrm{kg} / \mathrm{d} ;{ }^{\#} \mathrm{LT} 4 \pm 1 \mu \mathrm{g} / \mathrm{kg} / \mathrm{d}$

When TSH is increased, fT4 should be checked within 2-4 weeks along with a re-check of TSH. In case of increased TSH but normal fT4, watchful waiting can be adopted with measurement of TSH and fT4 every 6 weeks. In case of spontaneous TSH normalization, a 3-monthly screening of TSH is sufficient. In case of elevated TSH, if fT4 is decreased, or if symptoms/signs of hypothyroidism are present, or if baseline TPOAb status is positive, LT4 is indicated. If TSH is $>10 \mathrm{mU} / \mathrm{L}$, LT4 should be initiated irrespective of the levels of fT4 or antithyroid antibodies. The TSH level should be monitored every 6 weeks for as long as it remains abnormal, and the LT4 dose should be adapted accordingly. When TSH returns to normal under LT4 supplementation, one can proceed with TSH monitoring every 3 months.

If TSH is decreased, fT4 and TRAbs should be checked within 2 weeks along with a re-evaluation of TSH. A watchful waiting approach can be adopted in case of normal fT4 and absence of symptoms/signs of hyperthyroidism, with measurement of TSH and fT4 every 6 weeks. In case of spontaneous evolution towards normal TSH, 3-monthly screening of TSH is sufficient. In case of increased fT4 or symptoms/signs of hyperthyroidism, symptomatic treatment with propranolol should be started in case of hyperthyroid symptoms/signs or fT4 $>1.5$-fold the ULN. A thyroid 


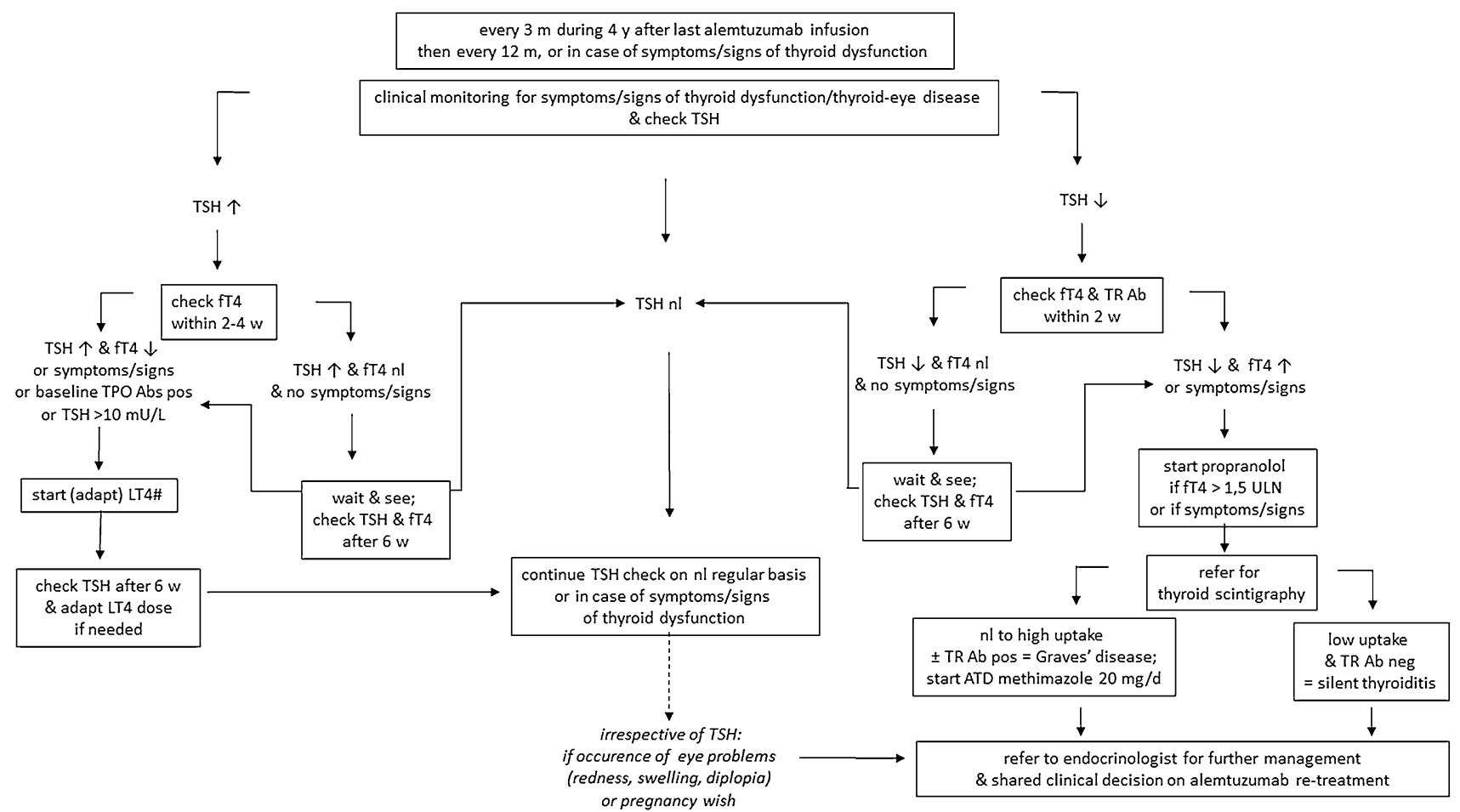

Fig. 2 Thyroid management algorithm post-alemtuzumab. $A b$ antibody, ATD anti-thyroid drug, fT4 free T4, LT4 levothyroxine, neg negative, $n l$ normal, pos positive, TPO thyroperoxidase, $T S H$ thyroid

scintigraphy represents the next step in the diagnostic workup. In case of low tracer uptake and negative TRAbs, the diagnosis of ST is most probable. In case of normal to high tracer uptake and positive TRAbs, the diagnosis of GD can be made, and treatment with an ATD (such as methimazole, thiamazole) can be started at a dose of $20 \mathrm{mg} / \mathrm{d}$ per os. In both cases (GD or ST), additional endocrinological advice is indicated with regard to further management.

In the occurrence of uni- or bilateral eye redness or swelling despite a normal TSH (expected to be very rare), the TRAbs should be checked and that patient should be referred to an endocrinologist.

\section{Pregnancy and thyroid disease in female patients treated with alemtuzumab}

Thyroid disease represents particular risks in women who are pregnant. Thyroid dysfunction during pregnancy increases the risk of miscarriage as well as obstetrical and fetal problems. In mothers with GD, maternal TRAbs can be transferred to the fetus, which can potentially result in transient fetal and neonatal hyperthyroidism. Placental transfer and potential pharmacologic activity of alemtuzumab were observed in mice during gestation and following delivery. Women of childbearing potential should use effective stimulating hormone, $T R$ thyrotropin receptor, $U L N$ upper limit of normal. ${ }^{\#} \mathrm{LT} 4 \pm 1 \mu \mathrm{g} / \mathrm{kg} / \mathrm{d}$, or $+25 \mu \mathrm{g} / \mathrm{d}$ in case of dose adaptation

contraceptive measures during treatment and for 4 months following a course of alemtuzumab [2].

Additionally, the Belgian taskforce proposes that female patients who want to become pregnant at any time after having been treated with alemtuzumab for MS irrespective of past thyroid function problems, serum TSH should be checked along with TPOAb and TRAb status and a clinical thyroid examination. In case of normal findings there is no contraindication for pregnancy from a thyroid point of view. However, in case one or more abnormal parameters are detected, or if the patient is currently receiving treatment for a thyroid problem, referral is indicated to an endocrinologist for advice with regard to thyroid-related pregnancy risk.

\section{Conclusions}

Available evidence suggests that in case of active MS, potential thyroid-related adverse events usually do not represent a contra-indication for treatment with alemtuzumab, as AITD most often has a mild clinical course. However, autoimmune TAEs occur frequently and necessitate patient education, careful clinical and biochemical monitoring, and adequate diagnostic and therapeutic action if thyroid dysfunction occurs. The proposed clinical management algorithm can serve as a tool in the daily clinical care for 
the alemtuzumab-treated MS patient. Beyond 4 years after the last alemtuzumab infusion we propose a lifelong yearly measurement of serum TSH as a minimal follow-up. Close interaction and collaboration is needed between the neurologist and endocrinologist, especially in case of (current or past) hyperthyroidism, GO, intolerance to ATDs, and in case of women seeking pregnancy. Finally, there is a need for more insight in the pathophysiology of alemtuzumabinduced thyroid dysfunction. Data on long-term outcomes in the clinical trials will certainly be of great help in the understanding of non-classical post-alemtuzumab AITD.

\section{Compliance with ethical standards}

Conflict of interest Author B.D. has received a speaker honorarium from Sanofi. Author E.B. has received consultancy honoraria from Biogen, Biologix, Genzyme, Medical Device Works, Merck Serono, Novartis, TEVA; has participated in local and international advisory boards of Biogen, Genzyme, Merck Serono, Novartis; has received travel grants from Bayer, Biogen, Biologix, Novartis. CHU Tivoli, La Louvière, has received research grants from Bayer, Biogen, Genzyme, Merck Serono, Novartis, TEVA. Author V.D. has received research grants from Biogen and Novartis, and has received a speaker honorarium from Biogen. Author S.E. has received travel grants from Bayer Schering, Biogen Idec, Merck-Serono, TEVA, Sanofi, and a consultancy honorarium from Bayer Schering, Merck-Serono, Sanofi. Author P.S. has received consultancy honorarium and travel grants from Biogen, Genzyme, Merck, Novartis, Roche, Schering, Serono and TEVA. Author B.V. has received research and travel grants, and speaker honoraria from Bayer-Schering, Biogen Idec, Sanofi Genzyme, MerckSerono, Novartis, Roche and TEVA. Author C.D. is a member of the executive committee of the European Group on Graves' Orbitopathy (EUGOGO). Editorial support was provided by Thomas Savoye, MD, Blue Jibe Medical Affairs, Herent, Belgium. Funding for this editorial support was provided by Sanofi. The manuscript was reviewed by Darren P. Baker PhD, Steve Cavalier MD, Madalina Chirieac MD, Andreas Lysandropoulos MD, Chantal Maertens PharmD PhD, and Isabel Firmino MD of Sanofi as a courtesy, with no obligation on the authors' part to adapt the manuscript.

Ethical approval This article does not contain any studies with human participants or animals performed by any of the authors.

Informed consent For this type of study, informed consent is not required.

Open Access This article is distributed under the terms of the Creative Commons Attribution 4.0 International License (http://creativeco mmons.org/licenses/by/4.0/), which permits unrestricted use, distribution, and reproduction in any medium, provided you give appropriate credit to the original author(s) and the source, provide a link to the Creative Commons license, and indicate if changes were made.

\section{References}

1. Berger T, Elovaara I, Fredrikson S et al (2017) Alemtuzumab use in clinical practice: recommendations from European Multiple Sclerosis Experts. CNS Drugs 31:33-50. https://doi.org/10.1056/ NEJMoa0802670
2. EMA—Lemtrada: EPAR_Product Information (2017) http:// www.ema.europa.eu/ema/index.jsp?curl=pages/medicines/human /medicines/003718/human_med_001678.jsp\&mid=WC0b01ac05 8001d124. Accessed 20 July 2017

3. CAMMS223 Trial Investigators, Coles AJ, Compston DA, Selmaj KW et al (2008) Alemtuzumab vs. interferon beta-1a in early multiple sclerosis. N Engl J Med 359(17):1786-1801. https://doi. org/10.1056/nejmoa0802670

4. Cohen JA, Coles AJ, Arnold DL, CARE-MS I investigators et al (2012) Alemtuzumab versus interferon beta 1a as first-line treatment for patients with relapsing-remitting multiple sclerosis: a randomised controlled phase 3 trial. Lancet 380:1819-1828. https ://doi.org/10.1016/S0140-6736(12)61769-3

5. Coles AJ, Twyman CL, Arnold DL, CARE-MS II investigators et al (2012) Alemtuzumab for patients with relapsing multiple sclerosis after disease-modifying therapy: a randomised controlled phase 3 trial. Lancet 380:1829-1839. https://doi. org/10.1016/S0140-6736(12)61768-1

6. Durelli L, De Mercanti SF, Cucci A et al (2016) Alemtuzumab long-lasting immunological effects: a 48 months follow-up observation. ECTRIMS Online Library, Sep 15, p. 146466

7. Ruck T, Bittner S, Wiendl H, Meuth SG (2015) Alemtuzumab in multiple sclerosis: mechanism of action and beyond. Int J Mol Sci 16:16414-16439. https://doi.org/10.3390/ijms160716414

8. Cox A, Thompson S, Jones J et al (2005) Lymphocyte homeostasis following therapeutic lymphocyte depletion in multiple sclerosis. Eur J Immunol 35:3332-3342. https://doi. org/10.1002/eji.200535075

9. Baker D, Herrod SS, Alvarez-Gonzalez C, Giovannoni G, Schmierer K (2017) Interpreting lymphocyte reconstitution data from the pivotal phase 3 trials of alemtuzumab. JAMA Neurol 74:961-969. https://doi.org/10.1001/jamaneurol.2017.0676

10. C Dayan, A Cuker, C LaGanke, on behalf of the CARE-MS Investigators et al (2016) Autoimmunity in patients treated With Alemtuzumab for relapsing-remitting multiple sclerosis: 5-year follow-up of the CARE-MS studies. ECTRIMS Online Library, Sep 16, p. 147015

11. Selmaj K, Habek M, Bass A et al (2017) Efficacy and safety of alemtuzumab in patients with RRMSIs durable over 10 years: follow-up from the CAMMS223 study. Neurology 88(16), Supplement P5.338

12. Havrdova E, Arnold D, Cohen J, Alemtuzumab CARE-MS I 5-year follow-up: Durable efficacy in the absence of continuous MS therapy et al (2017) Alemtuzumab CARE-MS I 5-year follow-up: Durable efficacy in the absence of continuous MS therapy. Neurology 89:1107-1116. https://doi.org/10.1212/ wnl.0000000000004313

13. Coles A, Cohen J, Fox E, CARE-MS II and CAMMS03409 Investigators et al (2017) Alemtuzumab CARE-MS II 5-year follow-up: Efficacy and safety findings. Neurology 89:11171126. https://doi.org/10.1212/wnl.0000000000004354

14. Antonelli A, Ferrari SM, Corrado A, Di Domenicantonio A, Fallahi P (2014) Autoimmune thyroid disorders. Autoimmun Rev 14:174-180. https://doi.org/10.1016/j.autrev.2014.10.016

15. Marrie RA, Reider N, Cohen J et al (2015) A systematic review of the incidence and prevalence of autoimmune disease in multiple sclerosis. Mult Scler 21:282-293. https://doi. org/10.1177/1352458514564490

16. Pearce SH, Brabant G, Duntas LH et al (2013) 2013 ETA guideline: management of subclinical hypothyroidism. Eur Thyroid J 2:215-228. https://doi.org/10.1159/000356507

17. Garber JR, Cobin RH, Gharib H, American Association Of Clinical Endocrinologists And American Thyroid Association Taskforce On Hypothyroidism In Adults et al (2013) Clinical practice guidelines for hypothyroidism in adults: cosponsored by the American Association of Clinical Endocrinologists and 
the American Thyroid Association. Thyroid 22:1200-1235. https://doi.org/10.1089/thy.2012.0205

18. Diana T, Krause J, Olivo PD et al (2017) Prevalence and clinical relevance of thyroid stimulating hormone receptor-blocking antibodies in autoimmune thyroid disease. Clin Exp Immunol 189:304-309. https://doi.org/10.1111/cei.12980

19. Biondi B, Bartalena L, Cooper DS et al (2015) The 2015 European Thyroid Association guidelines on diagnosis and treatment of endogenous subclinical hyperthyroidism. Eur Thyroid J 4:149-163. https://doi.org/10.1159/000438750

20. Ross DS, Burch HB, Cooper DS et al (2016) 2016 American Thyroid Association guidelines for diagnosis and management of hyperthyroidism and other causes of thyrotoxicosis. Thyroid 26:1343-1421. https://doi.org/10.1089/thy.2016.0229

21. Cooper DS (2005) Antithyroid drugs. N Engl J Med 352:905-917. https://doi.org/10.1056/NEJMra042972

22. Bartalena L, Baldeschi L, Boboridis K, European Group on Graves' Orbitopathy (EUGOGO) et al (2016) The 2016 European Thyroid Association/European Group on Graves' Orbitopathy guidelines for the management of Graves' orbitopathy. Eur Thyroid J 5:9-26. https://doi.org/10.1159/000443828

23. Pearce EN, Farwell AP, Braverman LE (2003) Thyroiditis. N Engl J Med 348:2646-2655. https://doi.org/10.1056/NEJMra021194

24. Poppe K, Velkeniers B, Glinoer D (2008) The role of thyroid autoimmunity in fertility and pregnancy. Nat Clin Pract Endocrinol Metab 4:394-405. https://doi.org/10.1038/ncpendmet0846

25. Alexander EK, Pearce EN, Brent GA et al (2017) Guidelines of the American Thyroid Association for the diagnosis and management of thyroid disease during pregnancy and the postpartum. Thyroid 27:315-389. https://doi.org/10.1089/thy.2016.0457

26. Mahzari M, Arnaout A, Freedman MS (2015) Alemtuzumab induced thyroid disease in multiple sclerosis: a review and approach to management. Can J Neurol Sci 42:284-291. https:// doi.org/10.1017/cjn.2015.48

27. Habek M, Arnold D, Cohen J et al (2012) Thyroid autoimmunity in comparison of alemtuzumab and Rebif ${ }^{\circledR}$ efficacy in multiple sclerosis studies I and II. In: Proceedings of the 22nd meeting of the European Neurological Society (ENS), vol 259

28. Coles AJ, Wing M, Smith S et al (1999) Pulsed monoclonal antibody treatment and autoimmune thyroid disease in multiple sclerosis. Lancet 354:1691-1695. https://doi.org/10.1016/S0140 $-6736(99) 02429-0$
29. Cossburn M, Pace AA, Jones J et al (2011) Autoimmune disease after alemtuzumab treatment for multiple sclerosis in a multicenter cohort. Neurology 77:573-579. https://doi.org/10.1212/ WNL.0b013e318228bec5

30. Senior PA, Arnold AL, Cohen JA et al (2016) Incidence and timing of thyroid adverse events in patients with RRMS treated with alemtuzumab. Neurology 86(16), Supplement P2.086

31. Daniels GH, Vladic A, Brinar V et al (2014) Alemtuzumab-related thyroid dysfunction in a phase 2 trial of patients with relapsingremitting multiple sclerosis. J Clin Endocrinol Metab 99:80-89. https://doi.org/10.1210/jc.2013-2201

32. Weetman AP (2014) Graves' disease following immune reconstitution or immunomodulatory treatment: should we manage it any differently? Clin Endocrinol 80:629-632. https://doi.org/10.1111/ cen. 12427

33. Rotondi M, Molteni M, Leporati P et al (2017) Autoimmune thyroid diseases in patients treated with alemtuzumab for multiple sclerosis: an example of selective anti-TSH-receptor immune response. Front Endocrinol (Lausanne). https://doi.org/10.3389/ fendo.2017.00254

34. Singer B, Hartung H, Margolin D et al (2015) Improvements in quality of life are maintained in alemtuzumab-treated RRMS patients who develop autoimmune adverse events: CARE-MS II. In: Annual meeting of the Consortium of Multiple Sclerosis Centers (CMSC)

35. Ziemssen T, Selmaj K, Havrdova E et al (2015) Quality-of-life improvements with alemtuzumab are maintained in relapsingremitting multiple sclerosis patients who develop thyroid adverse events in CARE-MS II. In: Meeting of the European Academy of Neurology (EAN)

36. Vermersch P, Giovannoni G, Cohen J et al (2015) Quality-of-life improvements in patients with active relapsing-remitting multiple sclerosis are not impacted by acute infections after receiving alemtuzumab in CARE-MS II. ECTRIMS Online Library, Oct 9, p. 115894

37. Wiersinga WM (2016) Clinical relevance of environmental factors in the pathogenesis of autoimmune thyroid disease. Endocrinol Metab (Seoul) 31:213-222. https://doi.org/10.3803/ EnM.2016.31.2.213 